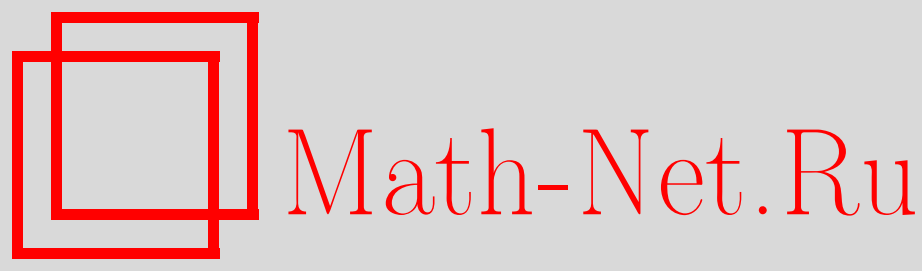

М. Д. Абловиц, Т. С. Хот, Связанные нелинейные уравнения Шредингера для соприкасающихся жидкостей со свободной поверхностью, ТМФ, 2009, том 159, номер 3, 326-335

DOI: https://doi.org/10.4213/tmf6353

Использование Общероссийского математического портала Math-Net.Ru подразумевает, что вы прочитали и согласны с пользовательским соглашением http: //www . mathnet.ru/rus/agreement

Параметры загрузки:

IP : 54.81 .137 .203

26 апреля 2023 г., 18:03:08

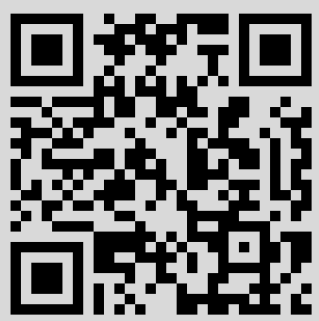




\title{
ФИЗИКА
}

Том 159, № 3

июнь, 2009

\section{СВЯЗАННЫЕ НЕЛИНЕЙНЫЕ УРАВНЕНИЯ ШРЕДИНГЕРА ДЛЯ СОПРИКАСАЮЩИХСЯ ЖИДКОСТЕЙ СО СВОБОДНОЙ ПОВЕРХНОСТЬЮ}

\begin{abstract}
Для соприкасающихся жидкостей со свободной поверхностью выведена новая система связанных нелинейных уравнений Шредингера в пределе квазимонохроматичности и малой амплитуды. При выводе использован недавно предложенный нелокальный формализм для описания соприкасающихся жидкостей, ограниченных свободной поверхностью.
\end{abstract}

Ключевые слова: жидкость с границей раздела, гидродинамика, нелинейная волна.

\section{1. ВВЕДЕНИЕ}

В настоящей работе мы выводим новую систему связанных нелинейных уравнений Шредингера (НУШ) для соприкасающихся жидкостей, разделенных свободной поверхностью.

Скалярное НУШ дает хорошо известное асимптотическое описание амплитуды модуляции квазимонохроматических волн. Это уравнение впервые было выведено для классических волн на воде в работе [1] с использованием гамильтонова подхода. Впоследствии в работе [2] было показано, что НУШ управляет амплитудой модуляции для продольно стратифицированных потоков (см. также [3] по поводу анализа модуляционной неустойчивости волн малой амплитуды). В работе [4] была выведена система связанных НУШ, асимптотически описывающая амплитудные модуляции квазимонохроматических волн с двумя пиками.

Мы рассмотрим две идеальные жидкости со свободной поверхностью раздела $\eta$, ограниченные свободной поверхностью $\beta+H$. В нашем выводе используется следующий интегральный формализм для описания соприкасающихся жидкостей со свободной поверхностью (см. вывод в работе [5]), который приводится в терминах свободной границы раздела $\eta$, свободной поверхности $\beta, q \equiv \varphi(\mathbf{x}, \eta, t), Q \equiv \Phi(\mathbf{x}, \eta, t)$

${ }^{*}$ Department of Applied Mathematics, University of Colorado at Boulder, USA. E-mail: terry.haut@colorado.edu 


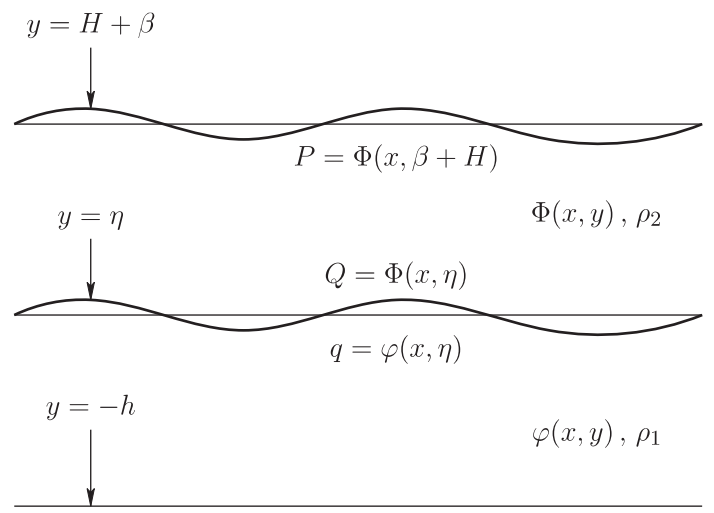

Две жидкости со свободной поверхностью и свободной границей раздела.

и $P \equiv \Phi(\mathbf{x}, \beta+H, t)$, где $\varphi$ и $\Phi-$ потенциалы скоростей нижней и верхней жидкостей (см. рисунок):

$$
\begin{aligned}
& \int_{\mathbb{R}^{2}} e^{-i \mathbf{k} \mathbf{x}^{\prime}} \operatorname{ch}(|\mathbf{k}| \eta+|\mathbf{k}| h) \eta_{t} d \mathbf{x}^{\prime}=i \int_{\mathbb{R}^{2}} e^{-i \mathbf{k} \mathbf{x}^{\prime}} \frac{\operatorname{sh}(|\mathbf{k}| \eta+|\mathbf{k}| h)}{|\mathbf{k}|}(\mathbf{k} \cdot \nabla) q d \mathbf{x}^{\prime}, \\
& \int_{\mathbb{R}^{2}} e^{-i \mathbf{k} \mathbf{x}^{\prime}} \operatorname{sh}(|\mathbf{k}| \beta) \beta_{t} d \mathbf{x}^{\prime}-\int_{\mathbb{R}^{2}} e^{-i \mathbf{k} \mathbf{x}^{\prime}} \operatorname{sh}(|\mathbf{k}|(\eta-H)) \eta_{t} d \mathbf{x}^{\prime}= \\
& =-i \int_{\mathbb{R}^{2}} e^{-i \mathbf{k} \mathbf{x}^{\prime}} \frac{\operatorname{ch}(|\mathbf{k}|(\eta-H))}{|\mathbf{k}|}(\mathbf{k} \cdot \nabla) Q d \mathbf{x}^{\prime}+ \\
& +i \int_{\mathbb{R}^{2}} e^{-i \mathbf{k} \mathbf{x}^{\prime}} \frac{\operatorname{ch}(|\mathbf{k}| \beta)}{|\mathbf{k}|}(\mathbf{k} \cdot \nabla) P d \mathbf{x}^{\prime}, \\
& \int_{\mathbb{R}^{2}} e^{-i \mathbf{k} \mathbf{x}^{\prime}} \operatorname{sh}(|\mathbf{k}|(\beta+H)) \beta_{t} d \mathbf{x}^{\prime}-\int_{\mathbb{R}^{2}} e^{-i \mathbf{k} \mathbf{x}^{\prime}} \operatorname{sh}(|\mathbf{k}| \eta) \eta_{t} d \mathbf{x}^{\prime}= \\
& =-i \int_{\mathbb{R}^{2}} e^{-i \mathbf{k} \mathbf{x}^{\prime}} \frac{\operatorname{ch}(|\mathbf{k}| \eta)}{|\mathbf{k}|}(\mathbf{k} \cdot \nabla) Q d \mathbf{x}^{\prime}+ \\
& +i \int_{\mathbb{R}^{2}} e^{-i \mathbf{k} \mathbf{x}^{\prime}} \frac{\operatorname{ch}(|\mathbf{k}|(\beta+H))}{|\mathbf{k}|}(\mathbf{k} \cdot \nabla) P d \mathbf{x}^{\prime}, \\
& P_{t}+\frac{1}{2}|\nabla P|^{2}+g \beta-\frac{\left(\beta_{t}+\nabla P \cdot \nabla \beta\right)^{2}}{2\left(1+|\nabla \beta|^{2}\right)}=\frac{\sigma_{2}}{\rho_{2}} \nabla \cdot\left(\frac{\nabla \beta}{\sqrt{1+|\nabla \beta|^{2}}}\right), \\
& \rho_{1}\left(q_{t}+\frac{1}{2}|\nabla q|^{2}+g \eta-\frac{\left(\eta_{t}+\nabla q \cdot \nabla \eta\right)^{2}}{2\left(1+|\nabla \eta|^{2}\right)}\right)- \\
& -\rho_{2}\left(Q_{t}+\frac{1}{2}|\nabla Q|^{2}+g \eta-\frac{\left(\eta_{t}+\nabla Q \cdot \nabla \eta\right)^{2}}{2\left(1+|\nabla \eta|^{2}\right)}\right)=\sigma_{1} \nabla \cdot\left(\frac{\nabla \eta}{\sqrt{1+|\nabla \eta|^{2}}}\right),
\end{aligned}
$$

a $\mathbf{k}=(k, l), \mathbf{x}=(x, y), \mathbf{x}^{\prime}=\left(x^{\prime}, y^{\prime}\right), \mathbf{k} \mathbf{x}^{\prime} \equiv \mathbf{k} \cdot \mathbf{x}^{\prime}, g$ - гравитационная постоянная, $\rho_{1}$, $\rho_{2}-$ плотность нижней и верхней жидкостей, $\sigma_{1}$ и $\sigma_{2}-$ поверхностное натяжение на 
границе раздела $\eta$ и на свободной поверхности $\beta+H$ соответственно. Уравнения (4) и (5) являются уравнениями Бернулли, записанными в терминах переменных границы раздела и поверхности $\eta, \beta, q, Q, P$. Интегральная формулировка (1)-(3) зависит от свободного спектрального параметра $k$ и называется в данной работе нелокальной спектральной формулировкой. Эта формулировка обобщает интегральный формализм классических волн на воде, полученный в работе [6].

Мы покажем, как использовать предположение о квазимонохроматичности и многомасштабный анализ нелокальной спектральной формулировки для вывода связанных НУШ. Другими словами, мы полагаем, что

$$
\begin{aligned}
& \eta(x, y, t)=e^{i\left(k_{1} x+l_{1} y-\omega_{1} t\right)} \eta_{1,0}(X, T)+e^{i\left(k_{2} x-\omega_{2} t\right)} \eta_{0,1}(X, T)+O(\epsilon), \\
& \beta(x, y, t)=e^{i\left(k_{1} x+l_{1} y-\omega_{1} t\right)} \beta_{1,0}(X, T)+e^{i\left(k_{2} x-\omega_{2} t\right)} \beta_{0,1}(X, T)+O(\epsilon)
\end{aligned}
$$

где $X=\epsilon x$ и $T=\epsilon t$. Тогда если взять $\xi=X-C_{\mathrm{g}_{1}} T$ (где $C_{\mathrm{g}_{1}}-$ групповая скорость), $\tau=\epsilon T, f(\xi, \tau)=\eta_{1,0}(X, T), g(\xi, \tau)=\beta_{0,1}(X, T)$, то получим следующие связанные уравнения:

$$
\begin{array}{r}
i f_{\tau}+\delta_{1} f_{\xi \xi}+\delta_{2}|f|^{2} f+\delta_{3}|g|^{2} f=0, \\
i g_{\tau}+i \Delta g_{\xi}+\delta_{4} g_{\xi \xi}+\delta_{5}|g|^{2} g+\delta_{6}|f|^{2} g=0,
\end{array}
$$

где

$$
\begin{array}{ll}
\delta_{1}=\frac{i\left(\gamma_{1}-\left(c_{1} / c_{2}\right) \gamma_{2}+\left(c_{1} / c_{2}\right)^{2} \gamma_{3}\right)}{c_{2}}, & \delta_{2}=\frac{i \gamma_{4}}{c_{2}}, \quad \delta_{3}=\frac{i \gamma_{5}}{c_{2}} \\
\delta_{4}=\frac{i\left(\alpha_{1}-\left(c_{1} / c_{2}\right) \alpha_{2}+\left(c_{1} / c_{2}\right)^{2} \alpha_{3}\right)}{c_{4}}, & \delta_{5}=\frac{i \alpha_{4}}{c_{4}},
\end{array}
$$

а коэффициенты $c_{1}, \ldots, c_{4}, \gamma_{1}, \ldots, \gamma_{5}$ и $\alpha_{1}, \ldots, \alpha_{5}$ зависят от $k_{1}, l_{1}, k_{2}, \omega_{1}, \omega_{2}$ и параметров поверхностных волн. При выводе требуется, чтобы групповая скорость $C_{\mathrm{g}_{1}}=c_{1} / c_{2}$ и $C_{\mathrm{g}_{2}}=c_{3} / c_{4}$ удовлетворяла соотношению

$$
C_{\mathrm{g}_{2}}\left(k_{2}\right)=C_{\mathrm{g}_{1}}\left(k_{1}, l_{1}\right)+\Delta \epsilon,
$$

где $\Delta$ - свободный параметр, появляющийся в уравнении (7). Мы проверили (численно), что при различных значениях $k_{1}$ и $l_{1}$ можно найти такое $k_{2}=k_{2}\left(k_{1}, l_{1}\right)$, что $C_{\mathrm{g}_{1}}=C_{\mathrm{g}_{2}}$. Поэтому $O(\epsilon)$-возмущение этого значения $k_{2}$ дает $C_{\mathrm{g}_{2}}=C_{\mathrm{g}_{1}}+\Delta \epsilon$ при подходящем $\Delta$.

Заметим, что $\delta_{1}=\omega_{1}^{\prime \prime} / 2$ и $\delta_{2}=\omega_{2}^{\prime \prime} / 2$ (дифференцирования по $k_{1}$ и $k_{2}$ соответственно). Хотя коэффициенты в уравнениях (6) и (7) слишком громоздкие (см. [7]), чтобы явно их здесь выписывать, мы покажем в следующем разделе, как вычислить их алгоритмически.

Подчеркнем особенность уравнений (6), (7), которая состоит в том, что их вывод требует размерности $2+1$. 


\section{2. ВЫВОД СВЯЗАННЫХ НУШ}

В данном разделе мы выведем уравнения (6), (7). Для этого предположим, что вектор

$$
\mathbf{u}(x, t)=(\eta(\mathbf{x}, t), \beta(\mathbf{x}, t), q(\mathbf{x}, t), Q(\mathbf{x}, t), P(\mathbf{x}, t))
$$

принимает вид

$$
\begin{aligned}
\mathbf{u}(\mathbf{x}, t) \sim & \left(e^{i \theta_{1}} \mathbf{u}_{1,0}(X, T)+e^{i \theta_{2}} \mathbf{u}_{0,1}(X, T)\right)+ \\
& +\epsilon\left(e^{2 i \theta_{1}} \mathbf{u}_{1,0}(X, T)+e^{2 i \theta_{2}} \mathbf{u}_{0,1}(X, T)+\mathbf{u}_{0,0}(X, T)+\right. \\
& \left.+e^{i\left(\theta_{1}+\theta_{2}\right)} \mathbf{u}_{1,1}(X, T)+e^{i\left(\theta_{1}-\theta_{2}\right)} \mathbf{u}_{1,-1}(X, T)\right)+ \text { c.c. }+\cdots
\end{aligned}
$$

где

$$
\begin{gathered}
\theta_{1}=k_{1} x+l_{1} y-\omega_{1} t, \quad \theta_{2}=k_{2} x-\omega_{2} t, \quad X=\epsilon x, \quad T=\epsilon T, \\
\mathbf{u}_{i, j}(X, T)=\left(\eta_{i, j}(X, T), \beta_{i, j}(X, T), q_{i, j}(X, T), Q_{i, j}(X, T), P_{i, j}(X, T)\right) .
\end{gathered}
$$

Другими словами, $\mathbf{u}_{m, n}(X, T)$ является (векторной) огибающей модуляции величин $e^{i m \theta_{1}}, e^{i n \theta_{2}}$.

После разложения уравнений Бернулли (1)-(5) по $\epsilon$ с использованием подстановки (8) соберем получающиеся выражения в гармоники $e^{i m \theta_{1}} e^{i n \theta_{2}}$, пренебрегая слагаемыми порядка $o\left(\epsilon^{2}\right)$. Полагая коэффициент при каждой такой гармонике равным нулю, с точностью до порядка $о\left(\epsilon^{2}\right)$ получаем уравнения, связывающие $\mathbf{u}_{m, n}(X, T)$, используя которые получаем уравнения (6), (7).

Перейдем к подробному выводу уравнений (6), (7). Мы разобьем этот вывод на следующие шесть шагов.

ШАГ 1. Разложим нелокальные спектральные уравнения (1)-(5) по $\epsilon$, пренебрегая членами порядка $о\left(\epsilon^{2}\right)$ :

$$
\begin{gathered}
\int_{\mathbb{R}^{2}} e^{-i \mathbf{k} \mathbf{x}^{\prime}}\left(\eta_{t}+\frac{i l \operatorname{th}(h|\mathbf{k}|) q_{y^{\prime}}}{|\mathbf{k}|}+\frac{i k \operatorname{th}(h|\mathbf{k}|) q_{x^{\prime}}}{|\mathbf{k}|}+\cdots\right) d \mathbf{x}^{\prime}=0 \\
\int_{\mathbb{R}^{2}} e^{-i \mathbf{k} \mathbf{x}^{\prime}}\left(\eta_{t}+\frac{i \operatorname{csch}(H|\mathbf{k}|) l P_{y^{\prime}}}{|\mathbf{k}|}-\frac{i \operatorname{cth}(H|\mathbf{k}|) l Q_{y^{\prime}}}{|\mathbf{k}|}+\right. \\
\left.+\frac{i \operatorname{csch}(H|\mathbf{k}|) k P_{x^{\prime}}}{|\mathbf{k}|}-\frac{i \operatorname{cth}(H|\mathbf{k}|) k Q_{x^{\prime}}}{|\mathbf{k}|}+\cdots\right) d \mathbf{x}^{\prime}=0 \\
\int_{\mathbb{R}^{2}} e^{-i \mathbf{k} \mathbf{x}^{\prime}}\left(\beta_{t}+\frac{i \operatorname{cth}(H|\mathbf{k}|) l P_{y^{\prime}}}{|\mathbf{k}|}-\frac{i \operatorname{csch}(H|\mathbf{k}|) l Q_{y^{\prime}}}{|\mathbf{k}|}+\right. \\
\left.+\frac{i \operatorname{cth}(H|\mathbf{k}|) k P_{x^{\prime}}}{|\mathbf{k}|}-\frac{i \operatorname{csch}(H|\mathbf{k}|) k Q_{x^{\prime}}}{|\mathbf{k}|}+\cdots\right) d \mathbf{x}^{\prime}=0 \\
g \beta+P_{t}-\frac{\sigma_{2} \beta_{y y}}{\rho_{2}}-\frac{\sigma_{2} \beta_{x x}}{\rho_{2}}+\cdots=0 \\
-\frac{\rho_{2} \eta}{\rho_{1}}+\eta+q_{t}-\frac{\rho_{2} Q_{t}}{\rho_{1}}-\frac{\sigma_{1} \eta_{y y}}{\rho_{1}}-\frac{\sigma_{1} \eta_{x x}}{\rho_{1}}+\cdots=0 .
\end{gathered}
$$


Для простоты мы не приводим явно вклады порядков $\epsilon$ и $\epsilon^{2}$, хотя и удерживаем их в разложениях.

ШАГ 2. Делая подстановку (8) в результат разложения уравнений Бернулли (12) и (13), соберем получающиеся уравнения в гармоники $e^{i m \theta_{1}} e^{i n \theta_{2}}, m, n=0,1,2$. Полагая коэффициент при каждой такой гармонике равным нулю, с точностью до порядка $o\left(\epsilon^{2}\right)$ получаем уравнения на переменные $\mathbf{u}_{m, n}(X, T)$, входящие в подстановку (8). Поскольку уравнения Бернулли - это дифференциальные уравнения в частных производных, этот шаг не представляет концептуальных сложностей.

С другой стороны, путем подстановки (8) в нелокальные уравнения (9)-(11) можно также собрать получающееся выражение в гармоники $e^{i m \theta_{1}}, e^{i n \theta_{2}}, m, n=0,1,2$, с точностью до порядка $o\left(\epsilon^{2}\right)$. А именно, после использования подстановки (8) в разложенных нелокальных уравнениях и взятия обратного преобразования Фурье получающихся выражений каждое из уравнений (9)-(11) принимает вид

$$
g(k, l) \int_{-\infty}^{\infty} \int_{-\infty}^{\infty} e^{-i k x^{\prime}-i l y^{\prime}} e^{i\left(k_{0} x+l_{0} y-\omega_{0} t\right)} F\left(\epsilon x^{\prime}, \epsilon t\right) d x^{\prime} d y^{\prime}+\cdots=0,
$$

где $k_{0}, l_{0}, \omega_{0}$ - вещественные числа, а опущенные слагаемые представляют собой слагаемые того же типа (заметим также, что мы заменили переменную интегрирования $x$ на $x^{\prime}$ ). Ниже мы покажем, что предыдущее уравнение можно записать как

$$
\begin{aligned}
e^{i\left(k_{0} x+l_{0} y-\omega_{0} t\right)}\left(g\left(k_{0}, l_{0}\right) F(X, T)\right. & -i \epsilon g_{k}\left(k_{0}, l_{0}\right) F_{X}(X, T)- \\
& \left.-\epsilon^{2} \frac{g_{k k}\left(k_{0}, l_{0}\right)}{2} F_{X X}(X, T)+\cdots\right)+\cdots=0 .
\end{aligned}
$$

Используем уравнение (15) в нелокальных уравнениях (9)-(11) и соберем результат в гармоники $e^{i m \theta_{1}} e^{i n \theta_{2}}, m, n=0,1,2$. Полагая коэффициенты каждой такой гармоники равными нулю с точностью до порядка $o\left(\epsilon^{2}\right)$, получаем оставшиеся уравнения на $\mathbf{u}_{m, n}(X, T)$.

Чтобы вывести уравнения (15), из (14) находим (опуская переменную $T$ ), что

$$
\begin{aligned}
g(k, l) & \int_{-\infty}^{\infty} \int_{-\infty}^{\infty} e^{-i k x^{\prime}-i l y^{\prime}} e^{i\left(k_{0} x+l_{0} y-\omega_{0} t\right)} F\left(\epsilon x^{\prime}\right) d x^{\prime} d y^{\prime}+\cdots= \\
& =e^{-i \omega_{0} t} g(k, l) \int_{-\infty}^{\infty} e^{-i x^{\prime}\left(k-k_{0}\right)} F\left(\epsilon x^{\prime}\right)\left(\int_{-\infty}^{\infty} e^{-i y^{\prime}\left(l-l_{0}\right)} d y^{\prime}\right) d x^{\prime}+\cdots= \\
& =e^{-i \omega_{0} t} \delta\left(l-l_{0}\right) g(k, l) \int_{-\infty}^{\infty} e^{-i x^{\prime}\left(k-k_{0}\right)} F\left(\epsilon x^{\prime}\right) d x^{\prime}+\cdots=0 .
\end{aligned}
$$

Теперь возьмем обратное преобразование Фурье последнего уравнения и получим

$$
\begin{aligned}
0 & =e^{-i \omega_{0} t} \int_{-\infty}^{\infty} \int_{-\infty}^{\infty} e^{i(k x+l y)} \delta\left(l-l_{0}\right) g(k, l) \int_{-\infty}^{\infty} e^{-i x^{\prime}\left(k-k_{0}\right)} F\left(\epsilon x^{\prime}\right) d x^{\prime} d l d k+\cdots= \\
& =e^{-i \omega_{0} t} \int_{-\infty}^{\infty} \int_{-\infty}^{\infty} e^{i k x} F\left(\epsilon x^{\prime}\right)\left(\int_{-\infty}^{\infty} \delta\left(l-l_{0}\right) e^{i l y} g(k, l) d l\right) e^{-i x^{\prime}\left(k-k_{0}\right)} d x^{\prime} d k+\cdots= \\
& =e^{-i \omega_{0} t} e^{i l_{0} y} \int_{-\infty}^{\infty} \int_{-\infty}^{\infty} e^{i k x} g\left(k, l_{0}\right) F\left(\epsilon x^{\prime}\right) e^{-i x^{\prime}\left(k-k_{0}\right)} d x^{\prime} d k+\cdots
\end{aligned}
$$


Далее сделаем замену переменных $X^{\prime}=\epsilon x^{\prime}, X=\epsilon x, K=\left(k-k_{0}\right) / \epsilon$. Тогда имеем

$$
\begin{aligned}
0 & =e^{i\left(l_{0} y-\omega_{0} t\right)} \int_{-\infty}^{\infty} e^{i\left(\epsilon K+k_{0}\right) x} g\left(k_{0}+\epsilon K, l_{0}\right) \int_{-\infty}^{\infty} e^{-i X^{\prime} K} F\left(X^{\prime}\right) d X^{\prime} d K+\cdots= \\
& =e^{i\left(k_{0} x+l_{0} y-\omega_{0} t\right)} \int_{-\infty}^{\infty} e^{i \epsilon K X} g\left(k_{0}+\epsilon K, l_{0}\right) \widehat{F}(K) d K+\cdots
\end{aligned}
$$

Наконец, разлагая $g\left(k_{0}+\epsilon K, l_{0}\right)$ вблизи $k_{0}$, получаем

$$
\begin{aligned}
0 & =e^{i\left(k_{0} x+l_{0} y-\omega_{0} t\right)} \int_{-\infty}^{\infty} e^{i \epsilon K X} g\left(k_{0}+\epsilon K, l_{0}\right) \widehat{F}(K) d K+\cdots= \\
& =e^{i\left(k_{0} x+l_{0} y-\omega_{0} t\right)} \int_{-\infty}^{\infty} e^{i \epsilon K X}\left(g\left(k_{0}, l_{0}\right)+\epsilon g_{k}\left(k_{0}, l_{0}\right) K+\cdots\right) \widehat{F}(K) d K+\cdots= \\
& =e^{i\left(k_{0} x+l_{0} y-\omega_{0} t\right)}\left(g\left(k_{0}, l_{0}\right) F(X)-i \epsilon g_{k}\left(k_{0}, l_{0}\right) F^{\prime}(X)+\cdots\right)+\cdots
\end{aligned}
$$

ШАГ 3. Получим связанные НУШ из коэффициентов при $e^{i \theta_{1}}$ и $e^{i \theta_{2}}$ в уравнениях (9)-(13). А именно, приравняем нулю коэффициенты при $e^{i \theta_{1}}$, получающиеся из уравнений (9)-(13):

$$
\begin{gathered}
-\kappa_{1} \operatorname{th}\left(h \kappa_{1}\right) q_{1,0}-i \omega_{1} \eta_{1,0}+\cdots=0, \\
-\operatorname{csch}\left(H \kappa_{1}\right) \kappa_{1} P_{1,0}+\operatorname{cth}\left(H \kappa_{1}\right) \kappa_{1} Q_{1,0}-i \omega_{1} \eta_{1,0}+\cdots=0, \\
-\operatorname{cth}\left(H \kappa_{1}\right) \kappa_{1} P_{1,0}+\operatorname{csch}\left(H \kappa_{1}\right) \kappa_{1} Q_{1,0}-i \omega_{1} \beta_{1,0}+\cdots=0 \\
\left(\frac{\sigma_{2} \kappa_{1}^{2}}{\rho_{2}}+g\right) \beta_{1,0}-i \omega_{1} P_{1,0}+\cdots=0, \\
-i \omega_{1} q_{1,0}+\frac{i \rho_{2} \omega_{1} Q_{1,0}}{\rho_{1}}+\frac{\left(\sigma_{1} \kappa_{1}^{2}+g \rho_{1}-g \rho_{2}\right) \eta_{1,0}}{\rho_{1}}+\cdots=0 .
\end{gathered}
$$

Аналогично приравняем нулю коэффициенты при $e^{i \theta_{2}}$, получающиеся из уравнений (9)-(13):

$$
\begin{gathered}
-k_{2} \operatorname{th}\left(h k_{2}\right) q_{0,1}-i \omega_{2} \eta_{0,1}+\cdots=0, \\
-\operatorname{csch}\left(H k_{2}\right) k_{2} P_{0,1}+\operatorname{cth}\left(H k_{2}\right) k_{2} Q_{0,1}-i \omega_{2} \eta_{0,1}+\cdots=0, \\
-\operatorname{cth}\left(H k_{2}\right) k_{2} P_{0,1}+\operatorname{csch}\left(H k_{2}\right) k_{2} Q_{0,1}-i \omega_{2} \beta_{0,1}+\cdots=0, \\
\left(\frac{\sigma_{2} k_{2}^{2}}{\rho_{2}}+g\right) \beta_{0,1}-i \omega_{2} P_{0,1}+\cdots=0, \\
-i \omega_{2} q_{0,1}+\frac{i \rho_{2} \omega_{2} Q_{0,1}}{\rho_{1}}+\frac{\left(\sigma_{1} k_{2}^{2}+g \rho_{1}-g \rho_{2}\right) \eta_{0,1}}{\rho_{1}}+\cdots=0 .
\end{gathered}
$$

Сделаем следующие подстановки в уравнения (16)-(19) и (21)-(24) соответственно:

$$
\begin{aligned}
& \mathbf{v}_{1,0}(X, T)=\mathbf{v}_{1,0,0}(X, T)+\epsilon \mathbf{v}_{1,0,1}(X, T)+\epsilon^{2} \mathbf{v}_{1,0,2}(X, T)+O\left(\epsilon^{3}\right), \\
& \mathbf{v}_{0,1}(X, T)=\mathbf{v}_{0,1,0}(X, T)+\epsilon \mathbf{v}_{0,1,0}(X, T)+\epsilon^{2} \mathbf{v}_{0,1,2}(X, T)+O\left(\epsilon^{3}\right),
\end{aligned}
$$


где мы определили

$$
\begin{aligned}
\mathbf{v}_{1,0}(X, T) & =\left(\beta_{1,0}(X, T), q_{1,0}(X, T), Q_{1,0}(X, T), P_{1,0}(X, T)\right), \\
\mathbf{v}_{1,0, j}(X, T) & =\left(\beta_{1,0, j}(X, T), q_{1,0, j}(X, T), Q_{1,0, j}(X, T), P_{1,0, j}(X, T)\right), \\
\mathbf{v}_{0,1}(X, T) & =\left(\eta_{0,1}(X, T), q_{0,1}(X, T), Q_{0,1}(X, T), P_{0,1}(X, T)\right), \\
\mathbf{v}_{0,1, j}(X, T) & =\left(\eta_{0,1, j}(X, T), q_{0,1, j}(X, T), Q_{0,1, j}(X, T), P_{0,1, j}(X, T)\right) .
\end{aligned}
$$

Далее положим коэффициенты при $\epsilon^{0}, \epsilon^{1}$ и $\epsilon^{2}$ равными нулю и итерационно решим уравнения относительно $\mathbf{v}_{1,0, j}(X, T)$ и $\mathbf{v}_{0,1, j}(X, T)$. Для коэффициентов при $\epsilon^{0}$ и $\epsilon^{1}$ это приводит к соотношениям

$$
\begin{aligned}
\mathbf{v}_{1,0,0}(X, T) & =\mathbf{a}_{1,0} \eta_{1,0}(X, T), \\
\mathbf{v}_{0,1,0}(X, T) & =\mathbf{a}_{0,1} \beta_{0,1}(X, T), \\
\mathbf{v}_{1,0,1}(X, T) & =\mathbf{b}_{1,0} \frac{\partial \eta_{1,0}}{\partial X}(X, T)+\mathbf{c}_{1,0} \frac{\partial \eta_{1,0}}{\partial T}(X, T), \\
\mathbf{v}_{0,1,0}(X, T) & =\mathbf{b}_{0,1} \frac{\partial \beta_{0,1}}{\partial X}(X, T)+\mathbf{c}_{0,1} \frac{\partial \beta_{0,1}}{\partial T}(X, T),
\end{aligned}
$$

где векторы $\mathbf{a}_{1,0}, \mathbf{a}_{0,1}, \mathbf{b}_{1,0}, \mathbf{c}_{1,0}, \mathbf{b}_{1,0}$ и $\mathbf{c}_{1,0}$ имеют вид

$$
\begin{array}{lll}
\mathbf{a}_{1,0}=\left(A_{1,0}\right)^{-1} \mathbf{a}_{1,0}^{\prime}, & \mathbf{a}_{0,1}=\left(A_{0,1}\right)^{-1} \mathbf{a}_{0,1}^{\prime}, & \mathbf{b}_{1,0}=\left(B_{1,0}\right)^{-1} \mathbf{b}_{1,0}^{\prime}, \\
\mathbf{c}_{1,0}=\left(B_{1,0}\right)^{-1} \mathbf{c}_{1,0}^{\prime}, & \mathbf{b}_{0,1}=\left(B_{0,1}\right)^{-1} \mathbf{b}_{0,1}^{\prime}, & \mathbf{c}_{0,1}=\left(B_{0,1}\right)^{-1} \mathbf{c}_{0,1}^{\prime} .
\end{array}
$$

Явный вид матриц $A_{1,0}, A_{0,1}, B_{1,0}$ и $B_{0,1}$, а также векторов $\mathbf{a}_{1,0}, \mathbf{a}_{0,1}, \mathbf{b}_{1,0}^{\prime}, \mathbf{c}_{1,0}^{\prime}$, $\mathbf{b}_{0,1}^{\prime}, \mathbf{c}_{0,1}^{\prime}$ приведен в работе [7]. Заметим, что векторы $\mathbf{b}_{1,0}^{\prime}, \mathbf{c}_{1,0}^{\prime}$ и $\mathbf{b}_{0,1}^{\prime}, \mathbf{c}_{0,1}^{\prime}$ зависят от $\mathbf{a}_{1,0}, \mathbf{a}_{0,1}$, поэтому $\mathbf{a}_{1,0}, \mathbf{a}_{0,1}$ следует вычислить сначала, а потом использовать в последующем вычислении $\mathbf{b}_{1,0}, \mathbf{c}_{1,0}$ и $\mathbf{b}_{0,1}=\mathbf{c}_{0,1}$.

Прежде чем найти решение для $\mathbf{v}_{0,1,2}(X, T)$ и $\mathbf{v}_{1,0,2}(X, T)$, надо выразить модуляции $\mathbf{u}_{2,0}(X, T), \mathbf{u}_{0,2}(X, T), \mathbf{u}_{1,1}(X, T), \mathbf{u}_{1,-1}(X, T)$ и $\mathbf{u}_{0,0}(X, T)$ в терминах $\eta_{1,0}(X, T)$ и $\beta_{0,1}(X, T)$ в главном порядке. Для этого нам требуются групповые скорости. Конкретно, подставим в (20) и (25) выражения

$$
\begin{aligned}
& \mathbf{v}_{1,0}(X, T)=\mathbf{v}_{1,0,0}(X, T)+\epsilon \mathbf{v}_{1,0,1}(X, T)+O\left(\epsilon^{2}\right), \\
& \mathbf{v}_{0,1}(X, T)=\mathbf{v}_{0,1,0}(X, T)+\epsilon \mathbf{v}_{0,1,0}(X, T)+O\left(\epsilon^{2}\right) .
\end{aligned}
$$

Это дает

$$
\begin{aligned}
& d_{1} \eta_{1,0}+\epsilon\left(c_{1} \frac{\partial \eta_{1,0}}{\partial X}+c_{2} \frac{\partial \eta_{1,0}}{\partial T}\right)=O\left(\epsilon^{2}\right), \\
& d_{2} \beta_{0,1}+\epsilon\left(c_{3} \frac{\partial \beta_{0,1}}{\partial X}+c_{4} \frac{\partial \beta_{0,1}}{\partial T}\right)=O\left(\epsilon^{2}\right) .
\end{aligned}
$$

Из выписанных уравнений видим, что групповые скорости $C_{\mathrm{g}_{1}}$ и $C_{\mathrm{g}_{2}}$ выражаются как $C_{\mathrm{g}_{1}}=c_{1} / c_{2}$ и $C_{\mathrm{g}_{2}}=c_{3} / c_{4}$. Коэффициенты $d_{1}, d_{2}$ и $c_{1}, \ldots, c_{4}$, возникающие в уравнениях (29), приведены в работе [7].

ШАГ 4. Теперь выразим модуляции $\mathbf{u}_{2,0}(X, T), \mathbf{u}_{0,2}(X, T), \mathbf{u}_{1,1}(X, T)$ и $\mathbf{u}_{1,-1}(X, T)$ в терминах $\eta_{1,0}(X, T)$ и $\beta_{0,1}(X, T)$ в главном порядке. Полагая коэффициенты при 
гармониках $e^{i\left(\theta_{1}+\theta_{2}\right)}, e^{i\left(\theta_{1}-\theta_{2}\right)}, e^{2 i \theta_{1}}$ и $e^{2 i \theta_{2}}$ равными нулю и пренебрегая членами порядка $O(\epsilon)$, находим, что модуляции $\mathbf{u}_{2,0}(X, T), \mathbf{u}_{0,2}(X, T), \mathbf{u}_{1,1}(X, T)$ и $\mathbf{u}_{1,-1}(X, T)$ можно выразить через $\eta_{1,0}(X, T)$ и $\beta_{0,1}(X, T)$ как

$$
\begin{aligned}
\mathbf{u}_{1,1}(X, T) & =\mathbf{a}_{1,1} \eta_{1,0}(X, T) \beta_{0,1}(X, T)+O(\epsilon), \\
\mathbf{u}_{1,-1}(X, T) & =\mathbf{a}_{1,-1} \eta_{1,0}(X, T) \overline{\beta_{0,1}}(X, T)+O(\epsilon), \\
\mathbf{u}_{2,0}(X, T) & =\mathbf{a}_{2,0} \eta_{1,0}(X, T)^{2}+O(\epsilon), \\
\mathbf{u}_{0,2}(X, T) & =\mathbf{a}_{0,2} \beta_{1,0}(X, T)^{2}+O(\epsilon) .
\end{aligned}
$$

Векторы $\mathbf{a}_{1,1}, \mathbf{a}_{1,-1}, \mathbf{a}_{2,0}$ и $\mathbf{a}_{0,2}$ имеют вид

$$
\begin{aligned}
& \mathbf{a}_{1,1}=\left(A_{1,1}\right)^{-1} \mathbf{a}_{1,1}^{\prime}, \quad \mathbf{a}_{1,-1}=\left(A_{1,-1}\right)^{-1} \mathbf{a}_{1,-1}^{\prime}, \\
& \mathbf{a}_{2,0}=\left(A_{2,0}\right)^{-1} \mathbf{a}_{2,0}^{\prime}, \quad \mathbf{a}_{0,2}=\left(A_{0,2}\right)^{-1} \mathbf{a}_{0,2}^{\prime},
\end{aligned}
$$

где матрицы $A_{1,1}, A_{1,-1}, A_{2,0}$ и $A_{0,2}$ и векторы $\mathbf{a}_{1,1}^{\prime}, \mathbf{a}_{1,-1}^{\prime}, \mathbf{a}_{2,0}^{\prime}$ и $\mathbf{a}_{0,2}^{\prime}$ приведены в работе [7]. Заметим, что они зависят от векторов $\mathbf{a}_{1,0}$ и $\mathbf{a}_{0,1}$, которые были получены на предыдущем шаге.

Аналогичным образом, полагая равными нулю коэффициенты при членах, зависящих только от медленных переменных, можно показать, что средние члены $\eta_{0,0}(X, T), \beta_{0,0}(X, T),\left(\partial q_{0,0} / \partial \xi\right)(X, T),\left(\partial Q_{0,0} / \partial \xi\right)(X, T)$ и $\left(\partial P_{0,0} / \partial \xi\right)(X, T)$ можно выразить через $\eta_{1,0}(X, T)$ и $\beta_{0,1}(X, T)$ как

$$
\begin{aligned}
\eta_{0,0}(X, T) & =\left(\mathbf{a}_{0,0}\right)_{1}\left|\eta_{1,0}(X, T)\right|^{2}+\left(\mathbf{a}_{0,0}\right)_{2}\left|\beta_{0,1}(X, T)\right|^{2}+O(\epsilon), \\
\beta_{0,0}(X, T) & =\left(\mathbf{a}_{0,0}\right)_{3}\left|\eta_{1,0}(X, T)\right|^{2}+\left(\mathbf{a}_{0,0}\right)_{4}\left|\beta_{0,1}(X, T)\right|^{2}+O(\epsilon), \\
\frac{\partial q_{0,0}}{\partial \xi}(X, T) & =\left(\mathbf{a}_{0,0}\right)_{5}\left|\eta_{1,0}(X, T)\right|^{2}+\left(\mathbf{a}_{0,0}\right)_{6}\left|\beta_{0,1}(X, T)\right|^{2}+O(\epsilon), \\
\frac{\partial Q_{0,0}}{\partial \xi}(X, T) & =\left(\mathbf{a}_{0,0}\right)_{7}\left|\eta_{1,0}(X, T)\right|^{2}+\left(\mathbf{a}_{0,0}\right)_{8}\left|\beta_{0,1}(X, T)\right|^{2}+O(\epsilon), \\
\frac{\partial P_{0,0}}{\partial \xi}(X, T) & =\left(\mathbf{a}_{0,0}\right)_{9}\left|\eta_{1,0}(X, T)\right|^{2}+\left(\mathbf{a}_{0,0}\right)_{10}\left|\beta_{0,1}(X, T)\right|^{2}+O(\epsilon),
\end{aligned}
$$

где $\mathbf{a}_{0,0}=\left(A^{0,0}\right)^{-1} \mathbf{a}_{0,0}^{\prime}$, а матрица $A_{0,0}$ и вектор $\mathbf{a}_{0,0}^{\prime}$ приведены в работе [7]. Заметим, что при получении выписанных выше уравнений для средних переменных мы использовали соотношения

$$
\begin{aligned}
& \partial_{T}\left|\eta_{1,0}(X, T)\right|^{2}=C_{\mathrm{g}_{1}} \partial_{X}\left|\eta_{1,0}(X, T)\right|^{2}+O(\epsilon), \\
& \partial_{T}\left|\beta_{0,1}(X, T)\right|^{2}=C_{\mathrm{g}_{2}} \partial_{X}\left|\beta_{0,1}(X, T)\right|^{2}+O(\epsilon),
\end{aligned}
$$

где групповые скорости удовлетворяют равенствам $C_{\mathrm{g}_{1}}=c_{1} / c_{2}$ и $C_{\mathrm{g}_{2}}=c_{3} / c_{4}$. Уравнения (30) следуют из уравнений (29), если положить $d_{1}=d_{2}=0$ и заметить, что групповые скорости являются вещественными.

ШАГ 5. Теперь найдем решение для $\mathbf{v}_{1,0,2}(X, T)$ и $\mathbf{v}_{0,1,2}(X, T)$ (напомним соотношения (28)) и используем выражения для $\mathbf{u}_{2,0}(X, T), \mathbf{u}_{0,2}(X, T), \mathbf{u}_{1,1}(X, T)$, $\mathbf{u}_{1,-1}(X, T)$ и $\mathbf{u}_{0,0}(X, T)$ в главном порядке, полученные на предыдущем шаге. Это 
дает

$$
\begin{aligned}
\mathbf{v}_{1,0,2}(X, T)= & \mathbf{d}_{1,0} \frac{\partial^{2} \eta_{1,0}}{\partial X^{2}}(X, T)+\mathbf{f}_{1,0} \frac{\partial^{2} \eta_{1,0}}{\partial X \partial T}(X, T)+\mathbf{m}_{1,0} \frac{\partial^{2} \eta_{1,0}}{\partial T^{2}}(X, T)+ \\
& +\mathbf{n}_{1,0}\left|\eta_{1,0}(X, T)\right|^{2} \eta_{1,0}(X, T)+\mathbf{r}_{1,0}\left|\beta_{1,0}(X, T)\right|^{2} \eta_{1,0}(X, T), \\
\mathbf{v}_{0,1,2}(X, T)= & \mathbf{d}_{0,1} \frac{\partial^{2} \beta_{0,1}}{\partial X^{2}}(X, T)+\mathbf{f}_{0,1} \frac{\partial^{2} \beta_{0,1}}{\partial X \partial T}(X, T)+\mathbf{m}_{0,1} \frac{\partial^{2} \beta_{0,1}}{\partial T^{2}}(X, T)+ \\
& +\mathbf{n}_{0,1}\left|\beta_{0,1}(X, T)\right|^{2} \beta_{0,1}(X, T)+\mathbf{r}_{0,1}\left|\eta_{1,0}(X, T)\right|^{2} \beta_{0,1}(X, T),
\end{aligned}
$$

где

$$
\begin{aligned}
& \mathbf{r}_{1,0}=\left(C_{1,0}\right)^{-1} \mathbf{r}_{1,0}^{\prime}, \quad \mathbf{d}_{1,0}=\left(C_{1,0}\right)^{-1} \mathbf{d}_{1,0}^{\prime}, \quad \mathbf{f}_{1,0}=\left(C_{1,0}\right)^{-1} \mathbf{f}_{1,0}^{\prime}, \\
& \mathbf{m}_{1,0}=\left(C_{1,0}\right)^{-1} \mathbf{m}_{1,0}^{\prime}, \quad \mathbf{n}_{1,0}=\left(C_{1,0}\right)^{-1} \mathbf{n}_{1,0}^{\prime}, \\
& \mathbf{c}_{0,1}=\left(C_{0,1}\right)^{-1} \mathbf{c}_{0,1}^{\prime}, \quad \mathbf{r}_{0,1}=\left(C_{0,1}\right)^{-1} \mathbf{r}_{0,1}^{\prime}, \quad \mathbf{f}_{0,1}=\left(C_{0,1}\right)^{-1} \mathbf{f}_{0,1}^{\prime}, \\
& \mathbf{m}_{0,1}=\left(C_{0,1}\right)^{-1} \mathbf{m}_{0,1}^{\prime}, \quad \mathbf{n}_{0,1}=\left(C_{0,1}\right)^{-1} \mathbf{n}_{0,1}^{\prime},
\end{aligned}
$$

а матрицы $C_{1,0}$ и $C_{0,1}$ и векторы $\mathbf{d}_{1,0}^{\prime}, \mathbf{f}_{1,0}^{\prime}, \mathbf{m}_{1,0}^{\prime}, \mathbf{r}_{1,0}^{\prime}, \mathbf{n}_{1,0}^{\prime}, \mathbf{d}_{0,1}^{\prime}, \mathbf{f}_{0,1}^{\prime}, \mathbf{m}_{0,1}^{\prime}, \mathbf{n}_{0,1}^{\prime}$ и $\mathbf{r}_{0,1}^{\prime}$ приведены в работе [7]. Заметим, что эти векторы зависят от векторов, вычисленных на предыдущих шагах.

ШАГ 6. Наконец, подставляя в уравнения (20) и (25) выражения (28), получаем

$$
\begin{aligned}
& d_{1} \eta_{1,0}+\epsilon\left(c_{1} \frac{\partial \eta_{1,0}}{\partial X}+c_{2} \frac{\partial \eta_{1,0}}{\partial T}\right)+\epsilon^{2}\left(\gamma_{1} \frac{\partial^{2} \eta_{1,0}}{\partial X^{2}}+\gamma_{2} \frac{\partial^{2} \eta_{1,0}}{\partial X \partial T}+\right. \\
&+\left.\gamma_{3} \frac{\partial^{2} \eta_{1,0}}{\partial T^{2}}+\gamma_{4}\left|\eta_{1,0}\right|^{2} \eta_{1,0}+\gamma_{5}\left|\beta_{1,0}\right|^{2} \eta_{1,0}\right)=O\left(\epsilon^{3}\right) \\
& d_{2} \beta_{0,1}+\epsilon\left(c_{3} \frac{\partial \beta_{0,1}}{\partial X}+c_{4} \frac{\partial \beta_{0,1}}{\partial T}\right)+\epsilon^{2}\left(\alpha_{1} \frac{\partial^{2} \beta_{0,1}}{\partial X^{2}}+\alpha_{2} \frac{\partial^{2} \beta_{0,1}}{\partial X \partial T}+\right. \\
&\left.+\alpha_{3} \frac{\partial^{2} \beta_{0,1}}{\partial T^{2}}+\alpha_{4}\left|\beta_{0,1}\right|^{2} \beta_{0,1}+\alpha_{5}\left|\eta_{1,0}\right|^{2} \beta_{0,1}\right)=O\left(\epsilon^{3}\right) .
\end{aligned}
$$

Коэффициенты $c_{1}, \ldots, c_{4}, \gamma_{1}, \ldots, \gamma_{5}$ и $\alpha_{1}, \ldots, \alpha_{5}$ приведены в работе [7].

Теперь мы готовы к выводу связанных НУШ из уравнений (32). Прежде всего, заметим, что можно найти такое решение для $\omega_{1}=\omega_{1}\left(k_{1}, l_{1}\right)$ и $\omega_{2}=\omega_{2}\left(k_{1}, k_{2}, l_{1}\right)$, для которого дисперсионные члены $d_{1}$ и $d_{2}$ обращаются в нуль. Далее, коэффициенты $c_{1}$ и $c_{2}$ зависят от $k_{1}$ и $l_{1}$, а коэффициенты $c_{3}$ и $c_{4}-$ от $k_{2}$. Для данного выбора $k_{1}$ и $l_{1}$ отношение $c_{1} / c_{2}$ фиксировано. Если можно найти такое $k_{2}=k_{2}\left(k_{1}, l_{1}\right)$, что $c_{3} / c_{4}=$ $c_{1} / c_{2}$, то можно заменой переменных получить связанные НУШ (мы проверили численно, что это можно сделать для целого ряда значений $k_{1}$ и $\left.l_{1}\right)$. В более общем случае, если

$$
\frac{c_{3}}{c_{4}}=\frac{c_{1}}{c_{2}}+\Delta \epsilon
$$

то запись уравнений (32) в терминах $\tau$ и $\xi$ дает уравнения (6), (7). 


\section{3. ЗАКЛЮЧЕНИЕ}

Новая система связанных НУШ выведена для соприкасающихся жидкостей со свободной поверхностью в пределе квазимонохроматичности и слабой нелинейности. Вывод основан на недавно предложенном нелокальном формализме для соприкасающихся жидкостей.

Благодарности. Настоящая работа частично поддержана со стороны NSF (грант DMS0604151).

\section{Список литературы}

[1] В. Е. Захаров, ПМТФ, 9:2 (1968), 86-94.

[2] R. Grimshaw, Wave Motion, 3:1 (1981), 81-103.

[3] R. H. J. Grimshaw, D. I. Pullin, J. Fluid Mech., 160 (1985), 297-315.

[4] G. J. Roskes, Phys. Fluids, 19:8 (1976), 1253-1254.

[5] T. S. Haut, M. J. Ablowitz, A reformulation and applications of interfacial fluids with a free surface, Preprint, Univ. Colorado, Boulder, 2008.

[6] M. J. Ablowitz, A. S. Fokas, Z. H. Musslimani, J. Fluid Mech., 562 (2006), 313-343.

[7] T.S. Haut, Integral formulations of water waves and multiple fluids: analysis and applications, PhD Thesis, Univ. Colorado, Boulder, 2008. 